\title{
DYNAMICAL SYSTEMS ANALYSIS OF A MODEL DESCRIBING TASMANIAN DEVIL FACIAL TUMOUR DISEASE
}

\author{
N. J. BEETON ${ }^{\bowtie 1}$ and L. K. FORBES ${ }^{2}$ \\ (Received 3 April, 2012; revised 23 September, 2012; first published online 18 March, 2013)
}

\begin{abstract}
A susceptible-exposed-infectious theoretical model describing Tasmanian devil population and disease dynamics is presented and mathematically analysed using a dynamical systems approach to determine its behaviour under a range of scenarios. The steady states of the system are calculated and their stability analysed. Closed forms for the bifurcation points between these steady states are found using the rate of removal of infected individuals as a bifurcation parameter. A small-amplitude Hopf region, in which the populations oscillate in time, is shown to be present and subjected to numerical analysis. The model is then studied in detail in relation to an unfolding parameter which describes the disease latent period. The model's behaviour is found to be biologically reasonable for Tasmanian devils and potentially applicable to other species.
\end{abstract}

2010 Mathematics subject classification: primary 92D25; secondary 34A34, 34D20, 34E10, 37M20, 37M05, 37G15.

Keywords and phrases: Tasmanian devil, epidemic model, nonlinear dynamics, stability, Hopf bifurcation.

\section{Introduction}

Coupled ordinary differential equation (ODE) systems are ubiquitous among epidemic models in use by ecologists. Susceptible-infectious-recovered (SIR) systems with mass-action (or density-dependent) transmission are used extensively in particular, but a host of variants are available in the literature, including susceptible-exposedinfectious-recovered (SEIR) and susceptible-infectious-susceptible (SIS) models. These are discussed in detail in a range of texts $[1,5,6,15]$ and have been used widely to model disease spread $[3,19]$. These models have necessarily become more detailed over time as basic assumptions, such as mass-action disease transmission [12] and

\footnotetext{
${ }^{1}$ School of Zoology, University of Tasmania, Sandy Bay, Tasmania 7005, Australia; e-mail: Nicholas.Beeton@utas.edu.au.

${ }^{2}$ School of Mathematics and Physics, University of Tasmania.

(C) Australian Mathematical Society 2013, Serial-fee code 1446-1811/2013\$16.00
} 
exponential growth without carrying capacity, have been found to be inadequate for many scenarios. A wealth of information can be gained from analysing such models: not only can these deterministic mechanistic models be solved and examined, but the stochastic models on which they are based can also be studied. Direct comparisons with real-life data, which is necessarily stochastic, can then be used to test the model assumptions [4, 7].

The basic reproduction number $R_{0}$, the expected number of secondary infectious cases per primary case in a naïve population, is a powerful way to distil the complexity of a dynamical model into a single number that is easy to calculate and tells us much about the expected behaviour of the system. However, things are not always so straightforward: Roberts [17] has pointed out that in many cases $R_{0}$ does not capture the whole story in disease dynamics. Even in single-species host-disease systems, multiple distinct equilibria are possible $[4,20]$. In this case, knowing whether $R_{0}>1$, and thus whether an epidemic will start, will not necessarily tell us the fate of the species.

Although these models are well understood and widely used, it is not always practicable for thorough dynamical systems analyses to be performed on all of their variants. While a more detailed analysis of a mechanistic modelling system may not seem to be necessary, it is important to understand the dynamics of the modelling system as unexpected model behaviour may exist. This may arise for one of two reasons. The first occurs when dynamics clearly do not represent the biological system correctly, generally due to the incorrect use of a modelling assumption or the use of an assumption in a situation for which it is invalid. The second is in the form of an emergent behaviour in the model that may result in unexpected but entirely plausible population dynamics. The use of dynamical systems analysis can thus give fresh insights into the behaviour of a biological system without necessarily requiring the collection and analysis of extensive field data.

The Tasmanian devil Sarcophilus harrisii is currently suffering from a unique and deadly infectious cancer known as devil facial tumour disease (DFTD) [11, 13]. The current evidence [14] suggests that the infection is transmitted via a frequency dependent process. This is unusual for a wildlife disease that is not sexually transmitted: most of these are assumed to have density dependent transmission, meaning that a population threshold exists under which the disease epidemic will die out [12]. Unfortunately, in the case of DFTD, it currently appears that no such threshold exists and the disease could persist until the host population is driven extinct. Also, unlike most wildlife diseases, DFTD is almost invariably fatal, meaning that the inclusion of a "recovered" class in the model is not necessary: any infectious animal will experience increased disease-induced mortality until it dies.

Modelling under these assumptions has recently been used in simulations to predict the potential effectiveness of disease suppression by removal (via culling) of infectious devils [4]. The Tasmanian devil-DFTD model has previously been studied along with a family of similar models: here we refer in particular to the work of Roberts and Jowett [18]. However, the model system is sufficiently different to those studied in 
the bulk of the literature that a thorough examination of its particular behaviour is worthwhile, especially considering the threatened status of the species [10] and the role that modelling has to play in its conservation. Studying the model framework may also prove useful for existing or future emerging frequency dependent diseases with a latent period.

In the present paper, we use dynamical systems theory to explore the behaviour of the host-disease population dynamics in the Tasmanian devil. We present this model in Section 2. We then look at the stability of the steady states and search for limit cycle solutions in Section 3. We perform a numerical analysis using an unfolding parameter in Section 4, and find a region in parameter space in which a Hopf bifurcation occurs and a small-amplitude limit cycle emerges. We then perform some numerical analysis in this region to explore the behaviour of the limit cycles. The results are discussed in Section 5 .

\section{Model selection}

2.1. Model description The model is a coupled ODE system with three components, namely the number of susceptible $(S)$, exposed $(E)$ and infectious $(I)$ Tasmanian devils, nondimensionalized such that the trapping region of the model for the total population $N$ lies between 0 and 1 . The unit $t$ measures time in years.

We assume logistic density dependence for fecundity, giving a susceptible birth rate of $b N(1-N)$ where $b$ is the maximum birth rate (occurring for a population near zero). A constant rate of mortality $\mu$ is assumed in each class, with an additional mortality rate $\alpha$ experienced by infectious animals. To simulate the effect of removing these animals, we also include a rate of removal effort $\rho$.

We assume that disease transmission occurs via a frequency dependent process [14]. This type of disease is modelled with a disease transmission function described by

$$
\frac{\beta S I}{N}
$$

which represents the rate of infection, where $\beta$ is the transmission coefficient. At the limit as $N, S$ and $I$ approach 0 , as would be expected in a population that is reaching extinction, the rate of infection is badly defined (has the form " $0 / 0$ "), and the behaviour of the system cannot be easily analysed at this point. To enable this analysis, we artificially introduce an asymptotically small parameter $\epsilon$ into the transmission function, changing it to

$$
\frac{\beta S I}{N+\epsilon} \text {. }
$$

Introducing this parameter makes some biological sense as we expect the infectiousness of the disease very near to $N=0$ to become identically zero, specifically, when there are either no susceptible or no infectious animals left in the population. Normally, in a deterministic setting, infection would continue in this case as "fractional" numbers of susceptible and infectious animals would remain. 
Once infected, devils are assumed to become infectious at a constant conversion rate $k$. The mean disease latent period $L$ can then be calculated as the inverse $(L=1 / k)$. This information gives the system of equations

$$
\begin{aligned}
& \frac{d S}{d t}=b N(1-N)-\mu S-\frac{\beta S I}{N+\epsilon}, \\
& \frac{d E}{d t}=\frac{\beta S I}{N+\epsilon}-(k+\mu) E, \\
& \frac{d I}{d t}=k E-(\mu+\alpha+\rho) I, \\
& N=S+E+I .
\end{aligned}
$$

All parameters are assumed positive except for the removal rate $\rho$. Although causing $\rho$ to become negative represents adding infectious animals to the population, a clearly unacceptable strategy, it is nevertheless explored here because this region exhibits interesting behaviour that may become relevant when the parameters are changed. In particular, the removal rate $\rho$ and mortality $\alpha$ are mathematically equivalent in this model: decreasing $\alpha$ has the same effect as decreasing $\rho$ and vice versa. As such, we can study changes in $\alpha$ in the system by instead studying a corresponding change in $\rho$. In this model, for example, a system with disease mortality $a$ and a negative removal rate $\rho=-c<0$ is equivalent to a system with a decreased disease mortality $a-c$ and zero removal rate $\rho=0$ : see point $\mathrm{C}$ in Figure 1 .

Such factors as disease evolution, host evolution or human interference could affect these and other parameters indirectly. However, we use $\rho$ as the bifurcation parameter in the analysis in Sections 3 and 4 as this parameter is the only one in the system that can be directly modified and controlled by human effort and is thus of most interest from a management perspective.

2.2. Model classification Roberts and Jowett [18] formulated a general form for SI and SEI models of an infectious disease in a wild animal population. Equations (2.1)-(2.4) above can be formulated as a specific case of the SEI framework described by Roberts and Jowett by setting their birth rate $B(N)$ to $b(1-N)$, death rate $D(N)$ to $\mu$, conversion rate $\sigma$ to $k$, disease mortality $\alpha$ to $\alpha+\rho$, contact rate $C(N)$ to $N /(N+\epsilon)$ and vertical transmission rate $p$ to zero.

The work avoids the previously mentioned issue around $N=0$ by changing the dynamic variables, or coordinates, of the system from $(S, E, I)$ to $(N, Y, Z)$, where $Y=E / N$ and $Z=I / N$. We can then take the limit of $\epsilon$ to zero, and thus $C(N)=1$.

They find that four steady states exist for any SEI system in this framework: a trivial solution where the population is unable to support itself, an equilibrium where disease is not present, one where the disease eliminates the host, and finally a steady state where disease is endemic. In Section 3 we confirm their result for our case in $(S, E, I)$ space, and further, in Section 4, demonstrate that the introduction of the asymptotic parameter $\epsilon$ to the model results in a small-amplitude limit cycle for a small region in 
the parameter space. This is absent in their model: Remark 7 of their paper shows that Hopf bifurcations do not exist for the system where $C(N)$ is constant.

\section{Dynamical systems analysis}

3.1. Finding the steady states We look for steady-state solutions for $(S, E, I)$ satisfying

$$
\frac{d S}{d t}=\frac{d E}{d t}=\frac{d I}{d t}=0
$$

We find, where $I \neq 0$, that $N$ satisfies a quadratic equation with solutions

$$
N=\frac{1}{2 b}\left(b-x y-\frac{x}{\beta}(\mu-x y)\right) \pm \frac{1}{2 b} \sqrt{\left(b-x y-\frac{x}{\beta}(\mu-x y)\right)^{2}-4 b \epsilon \frac{x}{\beta}(\mu-x y)},
$$

where for simplicity we define constants

$$
x=\frac{(k+\mu)(\mu+\alpha+\rho)}{k}, \quad y=\frac{k}{k+\mu+\alpha+\rho} .
$$

One solution to (3.1) is of order $N \sim 1$ and thus $\epsilon$-order terms can be excluded, giving

$$
N=1-\frac{(k+\mu)(\mu+\alpha+\rho)(\beta-\alpha-\rho)}{b \beta(k+\mu+\alpha+\rho)},
$$

which represents a steady state with a stable population with disease present. Nonzero populations in $S, E$ and $I$ can be described in terms of $N$.

The other solution to (3.1) is of order $N \sim \epsilon$, and we expand the square root in powers of $\epsilon$ using the binomial expansion $\sqrt{1+\epsilon}=(1+\epsilon / 2+\cdots)$, giving at first order

$$
N \approx \frac{x(x y-\mu)}{\beta(x y-b)-x(x y-\mu)} \epsilon,
$$

which represents, in the limit, a steady state with the population at extinction. The populations $S, E$ and $I$ are also of order $\epsilon$, and can be calculated in terms of $N+\epsilon$ (see equation (3.13) below).

Existence of two real solutions to the quadratic requires

$$
\left(b-x y-\frac{x}{\beta}(\mu-x y)\right)^{2} \geq 4 b \epsilon \frac{x}{\beta}(\mu-x y) .
$$

This will always be satisfied when the right-hand side of (3.4) is negative, which occurs when either

$$
\rho>-\alpha \text { or }-k-\mu-\alpha<\rho<-\mu-\alpha .
$$

In the case where the inequalities in (3.5) are not satisfied, as $\epsilon$ is arbitrarily small the existence condition reduces to

$$
\left(b-x y-\frac{x}{\beta}(\mu-x y)\right)^{2}>0 .
$$


So nonexistence is only possible in the region where the left-hand side of (3.6) is identically zero. This equation has roots at

$$
\rho=\mu+\alpha+\frac{-z \pm \sqrt{z^{2}-4 b k \beta(k+\mu)}}{2(k+\mu)},
$$

where we define

$$
z=\beta b-(\beta+\mu)(k+\mu) .
$$

We show later that these are the points where a transcritical bifurcation occurs between the two quadratic solutions of (3.1). The two roots in (3.7) can be shown to lie in the regions described in (3.5) for parameter values of interest, so both solutions of (3.1) exist for our purposes.

There are a further two solutions where $E=I=0$. In that case, $N=S$ and $N$ is then easily obtained from (2.1) to give

$$
N=0, \quad N=1-\frac{\mu}{b},
$$

which represent disease-free equilibrium states. In the former case the population is not viable even without the presence of disease and thus undergoes a "natural extinction", and in the latter a carrying capacity is reached.

Based on (3.9), (3.2) and (3.3) respectively, there are thus four steady states:

1. natural extinction,

$$
(S, E, I)=(0,0,0)
$$

2. disease extinction,

$$
(S, E, I)=(1-\mu / b, 0,0)
$$

3. coexistence,

$$
\begin{aligned}
(S, E, I)= & \left(\frac{x}{\beta}, \frac{\mu+\alpha+\rho}{k} y\left(1-\frac{x}{\beta}\right), y\left(1-\frac{x}{\beta}\right)\right) \\
& \times\left(1-\frac{(k+\mu)(\mu+\alpha+\rho)(\beta-\alpha-\rho)}{b \beta(k+\mu+\alpha+\rho)}\right)
\end{aligned}
$$

4. host extinction,

$$
(S, E, I)=\left(\frac{x}{\beta}, \frac{\mu+\alpha+\rho}{k} \frac{x y(b-\mu)}{\beta(x y-b)}, \frac{x y(b-\mu)}{\beta(x y-b)}\right)\left(\frac{x(x y-\mu)}{\beta(x y-b)-x(x y-\mu)}+1\right) \epsilon .
$$

3.2. Stability of the steady states The system of ODEs (2.1)-(2.4) is now linearized about each of the four steady states (3.10)-(3.13), as described in key texts such as those by Murray [15] and Edelstein-Keshet [6]. The behaviour of the solutions is 
determined by the eigenvalues of the Jacobian matrix for the ODE system, which has the general form

$$
\mathbf{J}=\left[\begin{array}{ccc}
b(1-2 N)-\mu-\frac{\beta I(N+\epsilon-S)}{(N+\epsilon)^{2}} & b(1-2 N)+\frac{\beta S I}{(N+\epsilon)^{2}} & b(1-2 N)-\frac{\beta S(N+\epsilon-I)}{(N+\epsilon)^{2}} \\
\frac{\beta I(N+\epsilon-S)}{(N+\epsilon)^{2}} & -\frac{\beta S I}{(N+\epsilon)^{2}}-(k+\mu) & \frac{\beta S(N+\epsilon-I)}{(N+\epsilon)^{2}} \\
0 & k & -(\mu+\alpha+\rho)
\end{array}\right] .
$$

This is now examined for each of the steady states in Section 3.1.

3.2.1. Natural extinction The eigenvalues of the Jacobian where $(S, E, I)=$ $(0,0,0)$ have negative real part if and only if $b<\mu$. This means that if the mortality $\mu$ outstrips the birth rate $b$, the population will be unsustainable and this extinction state will always be stable. However, this is not a scenario of interest to us here as we are interested in studying the effects of disease on an otherwise sustainable population. We henceforth assume that $b>\mu$, meaning that this solution is not of interest to us as a potential equilibrium despite playing an important role in dynamics in other disease models.

Roberts and Jowett [18] also assume that the birth rate at very low populations $B(0)$ is greater than the mortality rate $D(0)$, and then show that this equilibrium is always unstable in a more general setting.

3.2.2. Disease extinction For the second equilibrium point $S=1-(\mu / b), E=$ $I=0$ in equation (3.11), the Jacobian matrix (3.14) reduces to

$$
\mathbf{J}=\left[\begin{array}{ccc}
\mu-b & 2 \mu-b & 2 \mu-b-\beta \\
0 & -(k+\mu) & \beta \\
0 & k & -(\mu+\alpha+\rho)
\end{array}\right]
$$

with characteristic equation

$$
\operatorname{det}(\mathbf{J}-\lambda \mathbf{I})=(\mu-b-\lambda)[(k+\mu+\lambda)(\mu+\alpha+\rho+\lambda)-\beta k]=0 .
$$

Equation (3.15) is a cubic for $\lambda$, with the three solutions

$$
\begin{aligned}
\lambda_{1} & =\mu-b \\
\lambda_{2,3} & =\frac{1}{2}\left(-(2 \mu+\alpha+k+\rho) \pm \sqrt{(2 \mu+\alpha+k+\rho)^{2}-4[(k+\mu)(\mu+\alpha+\rho)-\beta k]}\right) .
\end{aligned}
$$

For a stable equilibrium, we require

$$
\rho>\frac{\beta k}{k+\mu}-\mu-\alpha, \quad \rho>-(2 \mu+\alpha+k), \quad b>\mu
$$

otherwise the equilibrium will be a saddle. 
A similar condition in the paper by Roberts and Jowett [18] requires the basic reproduction number $R_{0}$ to be less than one. In our case,

$$
R_{0}=\frac{k \beta}{(k+\mu)(\alpha+\rho+\mu)}
$$

and the first condition in (3.16) is equivalent to $R_{0}<1$. The third condition in (3.16) is satisfied by our previous assumption, and the second condition is always satisfied when $\rho>0$, which Roberts and Jowett [18] assume to be the case; although in our case, we allow $\rho$ to be negative for completeness.

For real values of $\lambda_{2,3}$ we require

$$
4[(k+\mu)(\mu+\alpha+\rho)-\beta k]<(2 \mu+\alpha+k+\rho)^{2} .
$$

We introduce $M=\mu+\alpha+\rho$, which represents the total mortality in the infectious $(I)$ compartment. Inequality (3.17) then simplifies to

$$
(M-\mu-k)^{2}>-4 \beta k .
$$

As $\beta$ and $k$ are both positive, this is always satisfied. All $\lambda$ are thus real, so the equilibrium is either a stable node or a saddle. No Hopf bifurcations can occur.

3.2.3. Coexistence For the third equilibrium point in equation (3.12), the Jacobian matrix $(3.14)$ reduces to

$$
\mathbf{J}=\left[\begin{array}{ccc}
B-\mu-\beta Z(1-X) & B+\beta X Z & B-\beta X(1-Z) \\
\beta Z(1-X) & -\beta X Z-(k+\mu) & \beta X(1-Z) \\
0 & k & -M
\end{array}\right],
$$

where we define variables $X=S /(N+\epsilon), Z=I /(N+\epsilon)$ and $B=b(1-2 N)$. This gives the characteristic equation

$$
\begin{gathered}
(B-\mu-\beta Z(1-X)-\lambda)[(\beta X Z+k+\mu+\lambda)(M+\lambda)-k \beta X(1-Z)] \\
\quad+\beta Z(1-X)[k(B-\beta X(1-Z))+(M+\lambda)(B+\beta X Z)]=0
\end{gathered}
$$

for the eigenvalues $\lambda$.

We are interested in knowing the stability of this equilibrium in terms of the bifurcation parameter $\rho$. A necessary but not sufficient condition for a change in stability is the real component of any solution of (3.19) changing sign. In our case, where the characteristic equation is a cubic and there are thus three solutions for $\lambda$, this can happen in one of two ways:

One of the solutions for $\lambda$ is identically zero or undergoes a sign-changing singularity, that is,

$$
\lambda=0
$$

or a complex conjugate pair of solutions for $\lambda$ both have real part zero, namely

$$
\lambda=0 \pm \beta i
$$


In the first case, where (3.20) is satisfied, equation (3.19) further simplifies to

$$
\begin{aligned}
& (B-\mu-\beta Z(1-X))[M(\beta X Z+k+\mu)-k \beta X(1-Z)] \\
& \quad+\beta Z(1-X)[k(B-\beta X(1-Z))+M(B+\beta X Z)]=0 .
\end{aligned}
$$

The variables $B, X$, and $Z$ can be written in terms of $M$, which in turn can be written in terms of $\rho$. Substituting in values from (3.12) and rearranging equation (3.22) in terms of $M$ gives a rational function with a cubic as the numerator:

$$
\frac{(k(M-\beta)+M \mu)\left[M(b \beta+(M-\beta-\mu) \mu)+k\left(M^{2}+b \beta-M(\beta+\mu)\right)\right]}{(k+M) \beta}=0 .
$$

Equation (3.23) has roots at

$$
M=\frac{-z \pm \sqrt{z^{2}-4 b k \beta(k+\mu)}}{2(k+\mu)}, \quad M=\frac{\beta k}{k+\mu},
$$

where $z$ is as previously defined in equation (3.8).

The characteristic equation (3.23) also has a singularity at $M=-k$, which represents an additional potential change in stability. As $k$ is positive, and $\beta$ and $\mu$ are nonnegative, it follows that

$$
-k<\frac{\beta k}{k+\mu} \text {. }
$$

So the solution at $-k$ always occurs at a smaller value of $\rho$ than the solution at $\beta k /(k+\mu)$.

The first condition in (3.24) has two real solutions when

$$
z^{2}-4 b k \beta(k+\mu)>0 .
$$

This inequality can be re-expressed as a quadratic in an unfolding parameter $k$. We choose this parameter as it is the least well known biologically and has been shown previously to have an important effect on disease dynamics [4]. Condition (3.25) is obeyed for either of

$$
\begin{aligned}
& k<\frac{\beta b}{(\beta+\mu)^{2}-4 \beta b}((\beta-\mu)-\sqrt{4 \beta(b-\mu)})-\mu, \\
& k>\frac{\beta b}{(\beta+\mu)^{2}-4 \beta b}((\beta-\mu)+\sqrt{4 \beta(b-\mu)})-\mu .
\end{aligned}
$$

The regions specified in (3.26) and (3.27) describe two branches of the first condition in (3.24). The first branch is equivalent to the condition defined by Robert and Jowett [18] as $R_{2}>1$. The other branch is ignored in their analysis as it does not represent a change in stability, as we verify visually later in Figure 1 . The condition $M=-k$ is also ignored in their analysis as it requires $\rho$ to be negative. The remaining condition $M=(\beta k) /(k+\mu)$ describes a change in stability from case 3 to case 2 . 
We confirm later numerically that where the inequalities (3.26) and (3.27) are satisfied, this solution is stable for either of

$$
\begin{aligned}
& -k-\mu-\alpha<\rho<\frac{-z-\sqrt{z^{2}-4 b k \beta(k+\mu)}}{2(k+\mu)}-\mu-\alpha, \\
& \frac{-z+\sqrt{z^{2}-4 b k \beta(k+\mu)}}{2(k+\mu)}-\mu-\alpha<\rho<\frac{\beta k}{k+\mu}-\mu-\alpha,
\end{aligned}
$$

and where (3.26) and (3.27) are not satisfied, it is stable for

$$
-k-\mu-\alpha<\rho<\frac{\beta k}{k+\mu}-\mu-\alpha .
$$

In the second case where a stability change is possible, a complex conjugate pair of eigenvalues with real part zero exists, as per (3.21). In this case, (3.19) can be expressed as

$$
(\lambda+x)(\lambda-\beta i)(\lambda+\beta i)=0
$$

We expand (3.28), giving

$$
\lambda^{3}+x \lambda^{2}+\beta^{2} \lambda+x \beta^{2}=0
$$

For a change in stability to occur, the real eigenvalue must be negative, and we thus require $x$ positive. This is a Hopf bifurcation and, for a characteristic equation of the general form $\lambda^{3}+a_{1} \lambda^{2}+a_{2} \lambda+a_{3}=0$, the required conditions are

$$
a_{1}>0, \quad a_{2}>0, \quad a_{3}=a_{1} a_{2} .
$$

We can solve for $M$ for all three conditions in (3.29) to determine if-and, if so, where-a Hopf bifurcation occurs. After removing the singularity at $M=-k$, the first condition $a_{1}>0$ becomes a quadratic in $M$ :

$$
2(k+\mu) M^{2}+(1+\beta(b-2 k)-2 \mu(k+\mu)) M+\beta k(\beta+b+k+2 \mu)=0 .
$$

Similarly, the second condition $a_{2}>0$ becomes a quartic in $M$. The third condition $a_{3}=a_{1} a_{2}$ becomes a quintic in $M$. As these conditions are too complicated to calculate analytically, this problem is left for numerical analysis in Section 4.

3.2.4. Host extinction For the equilibrium in equation (3.13), we have the same characteristic equation as in case 3 (equation (3.22)). In this case, $X$ and $Z$ take the values

$$
X=\frac{x}{\beta}, \quad Z=\frac{x y(b-\mu)}{\beta(x y-b)} .
$$

As $N \sim \epsilon$, it follows that $N \ll 1$, so $B \approx b$. 
Solving where (3.20) is satisfied, as in case 3, gives

$$
\begin{aligned}
& \frac{M\left[(b-\mu)(k+\mu)^{2} M^{2}+(b-\mu)(k+\mu)(b \beta-(k+\mu)(\beta+\mu)) M+b k \beta(b-\mu)(k+\mu)\right]}{\beta(M(k+\mu)-b(k+M))} \\
& \quad=0 .
\end{aligned}
$$

Equating the numerator of equation (3.31) to zero gives the solutions

$$
\begin{aligned}
& \rho=-\mu-\alpha, \\
& \rho=\frac{-z \pm \sqrt{z^{2}-4 b k \beta(k+\mu)}}{2(k+\mu)}-\mu-\alpha,
\end{aligned}
$$

where $z$ is again as defined in equation (3.8). In addition, the denominator can also become zero, and this gives the additional solution

$$
\rho=-\frac{b k}{b-k-\mu}-\mu-\alpha .
$$

It is shown later numerically that the solutions (3.32) and (3.33) do not represent an overall change in stability of the equilibrium. As mentioned above, the change of sign of the eigenvalue represents a change in stability of only one of the eigenvectors. In these cases the other two eigenvectors have opposing stability (one is stable and the other is unstable), meaning that the stability of the third eigenvector is irrelevant.

This solution is thus stable for

$$
\frac{-z-\sqrt{z^{2}-4 b k \beta(k+\mu)}}{2(k+\mu)}-\mu-\alpha<\rho<\frac{-z+\sqrt{z^{2}-4 b k \beta(k+\mu)}}{2(k+\mu)}-\mu-\alpha,
$$

where the square root terms in (3.34) are real. The regions in which this occurs are given above in the analysis for case 3 .

\section{Numerical analysis}

The conditions calculated analytically in the previous section include some where no change in stability actually occurs, and do not include the possibility of Hopf bifurcations. To gain a more complete picture of the behaviour of the system, we need to perform a numerical analysis. All analyses and figures were created using the $\mathrm{R}$ programming language (version 2.13) [16].

4.1. Model parameters In Figure 1, we explore a cross-section of parameter space using an unfolding parameter diagram. In addition to our bifurcation parameter $\rho$, we vary the mean latent period (expressible as $L=1 / k$ ) and calculate the stability of our three equilibria directly at each point in the diagram by calculating the eigenvalues and eigenvectors of the Jacobian matrix over a range of values for both of these parameters.

Mortality, fecundity, disease latent period and disease mortality are set at $\mu=0.25 \log (2)$ years $^{-1}, b=\mu+\log (1.25)$ years $^{-1}, L=6$ months $\left(k=2\right.$ years $\left.^{-1}\right)$ 
and $\alpha=4$ years $^{-1}$ (mortality in 3 months on average), respectively, based on observations of the Tasmanian devil and DFTD [4]. The remaining free parameter, $\beta$, can be derived from these.

The biological variable $r_{0}$ represents the initial increase of disease prevalence in a naïve population in the absence of experimental manipulation (as for case 2 but with small but positive $E$ and $I$, with $\rho=0$ ). It is mathematically defined as

$$
r_{0}=\lim _{P \rightarrow 0} \frac{1}{P} \frac{d P}{d t} .
$$

An estimate for $r_{0}$ is available based on observations made at the Forestier peninsula, and has been calculated at 2.2644 years $^{-1}$ [14]. In our model, disease prevalence can be defined as $P=I / N$, and so $r_{0}$ can be defined in terms of model variables using (2.1)-(2.4):

$$
\begin{aligned}
r_{0} & =\lim _{P \rightarrow 0}\left(\frac{1}{I} \frac{d I}{d t}-\frac{1}{N} \frac{d N}{d t}\right) \\
& =\frac{k E}{I}-(\mu+\alpha)-\left[b\left(1-\left(1-\frac{\mu}{b}\right)\right)-\mu\right]=\frac{k E}{I}-(\mu+\alpha) .
\end{aligned}
$$

As $E$ and $I$ are approximately zero in a naïve population, we take the limit of their quotient:

$$
\lim _{P \rightarrow 0} \frac{E}{I}=\lim _{P \rightarrow 0} \frac{\frac{d E}{d t}}{\frac{d I}{d t}}=\lim _{P \rightarrow 0} \frac{\beta I-(k+\mu) E}{k E-(\mu+\alpha) I}=\lim _{P \rightarrow 0} \frac{\beta-(k+\mu) \frac{E}{I}}{k \frac{E}{I}-(\mu+\alpha)} .
$$

We then rearrange for $\beta$ :

$$
\beta=\lim _{P \rightarrow 0} \frac{E}{I}\left(k \frac{E}{I}+(k-\alpha)\right) .
$$

Rearranging equation (4.1) solves for $\lim _{P \rightarrow 0} E / I$ in terms of $r_{0}$ and the other model parameters. Setting these parameters to the given values yields a value for $\beta$ of approximately 14.28 years $^{-1}$, using equation (4.2).

We henceforth assume that disease infectiousness $\beta$ remains constant at this value as we vary $k$ and $\rho$. However, as shown in (4.2), $\beta$ is dependent on the conversion rate $k$ and disease mortality $\alpha$. The results here therefore indicate the effects of a disease with constant infectiousness but varying latent period and thus, from (4.1), a varying increase in prevalence $r_{0}$. Conversely, Beeton and McCallum [4] assume $r_{0}$ to be constant based on the known measurement, and the disease infectiousness $\beta$ is allowed to vary with $k$.

4.2. Results We find that at no point is more than one equilibrium stable. There are thus four potential states for the system:

- unstable-no equilibrium is stable;

- host extinction-case 4 is stable;

- coexistence-case 3 is stable;

- disease extinction-case 2 is stable. 


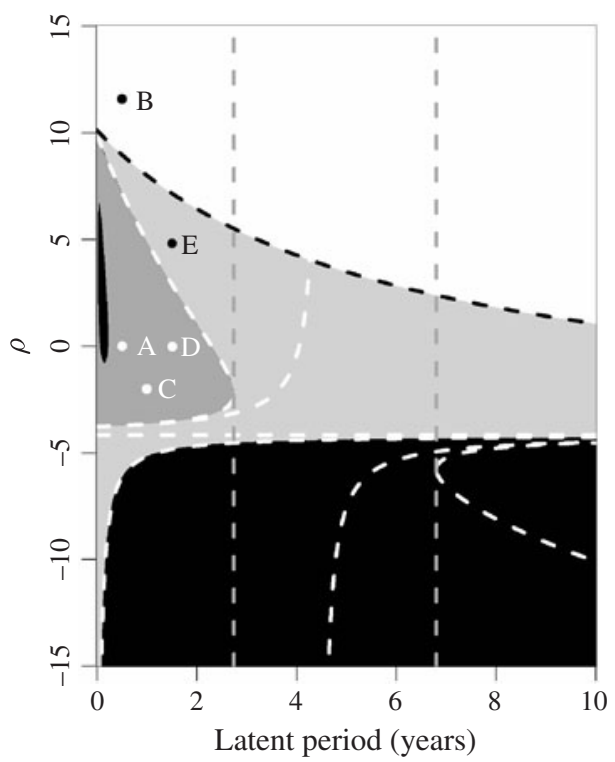

FIGURE 1. Unfolding parameter diagram. Shading denotes regions where a different solution (host extinction is shaded dark grey, coexistence light grey, and disease extinction white) or no solution (black) is stable. The black and white dashed curves show all locations of potential changes in stability, calculated analytically. The grey vertical dashed lines show the values of $L$ between which an extinction state is not possible analytically. Point A represents a current estimate of parameters for the devil-DFTD system; point B represents a removal effort equivalent to removing $95 \%$ of infected devils every 3 months (or $\rho \approx 11.98$ ); point $\mathrm{C}$ represents a slower growing, less deadly disease (latent period of 1 year, $\rho=-2$ ); point D represents a very slow growing disease (latent period of 18 months); and point E represents that disease with a corresponding quarterly $70 \%$ removal effort.

Figure 1 confirms that the solutions (3.32) and (3.33), despite being flagged in our previous analysis, do not affect the stability of the equilibrium described by (3.13): these correspond to the two white dashed curves within the coexistence region. It can also be seen that the right branch of the extinction state is also not stable despite being taken into account analytically.

We take two biologically interesting cross-sections of Figure 1 to show the equilibrium behaviour of the system in more detail. The first cross-section we take is at $k=2$ years $^{-1}$, or where the latent period $L$ of the disease is 6 months. This represents the current best guess of the latent period of the disease [4].

The bifurcation diagram based on this cross-section is shown in Figure 2. For realistic values of removal effort, that is, nonnegative values of $\rho$, we see that increasing effort can cause a transcritical bifurcation at two points, the first being between host extinction and coexistence (at about $\rho=7.2$ years $^{-1}$ ), and the second between coexistence and disease extinction (at $\rho=9.0$ years $^{-1}$ ). Interestingly, decreasing $\rho$ below zero also causes a transcritical bifurcation between host extinction and coexistence, and this is visible at about $\rho=-3.7$ years $^{-1}$. However, further decreasing $\rho$ beyond this point cannot cause disease extinction; instead, there exists 


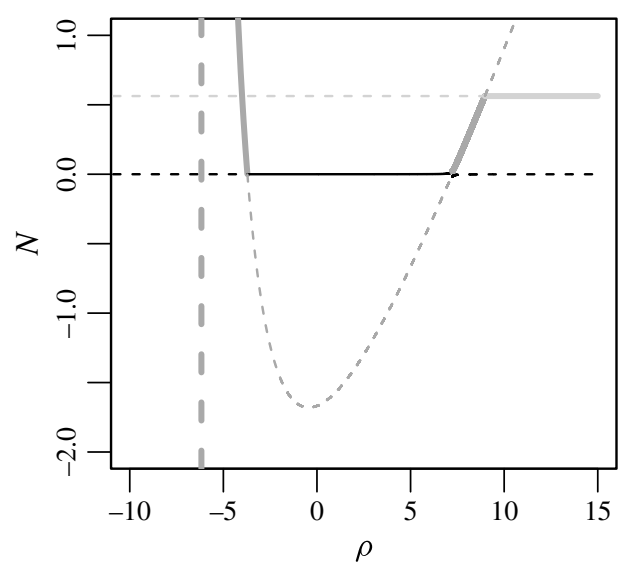

FIGURE 2. Bifurcation diagram at $L=6$ months. Thick solid lines denote a stable equilibrium and thin dashed lines represent an unstable equilibrium. The colours of these lines denote distinct equilibria (black is host extinction, dark grey coexistence, and light grey disease extinction). The thick dashed vertical line shows the value for $\rho$ below which there is no stable equilibrium.

a threshold beyond which no solution is stable and the system is divergent. The biological assumptions in the model are violated and the system thus becomes biologically invalid beyond this threshold. This occurs in Figure 2 for $\rho<$ -6.2 years $^{-1}$. The unstable equilibrium displayed in the figure at negative values of $N$ also represents an obvious violation of the biological assumptions; however, due to the existence of a trapping region for $N \geq 0$ when $b>\mu$, this region of the state space will never be reached.

In the far left of Figure 1, a region of instability can be seen displayed in black (at $L \approx 0.05-0.20$ years, $\rho \approx 0-6$ years $^{-1}$ ) which was not calculated in Section 3 and is not marked by dashed lines, although the borders of this region can be predicted from the Hopf analysis in (3.29). As we have calculated all possible changes in stability where (3.20) is satisfied, this change in stability is necessarily due to (3.21) being satisfied. The boundary of this region therefore represents a Hopf bifurcation, and a limit cycle exists inside this region. We set our second vertical cross-section to cut through the maximum-amplitude part of the Hopf region to see limit cycle behaviour by choosing $L \approx 22$ days $(L \approx 0.05$ years in Figure 1$)$. We here and henceforth set $\epsilon=10^{-4}$ so that this small-amplitude limit cycle behaviour is visible in our numerical analyses.

The bifurcation diagram based on this cross-section is shown in Figure 3. A logarithmic scale has been chosen in order to highlight the small-amplitude behaviour of the system. Ignoring these small-amplitude behaviours for a moment, the same behaviours that were seen in Figure 2 are evident here in that similar transcritical bifurcations occur (at about $\rho=-3.8,9.5$ and 10.0 years $^{-1}$ ). However, we see that the total population $N$ never quite reaches zero, but instead is of order $\epsilon$ where the host extinction scenario is stable. A limit cycle emerges in the middle of this scenario through Hopf bifurcations at about $\rho=0.1$ years $^{-1}$ and again at $\rho=6.7$ years $^{-1}$, but 


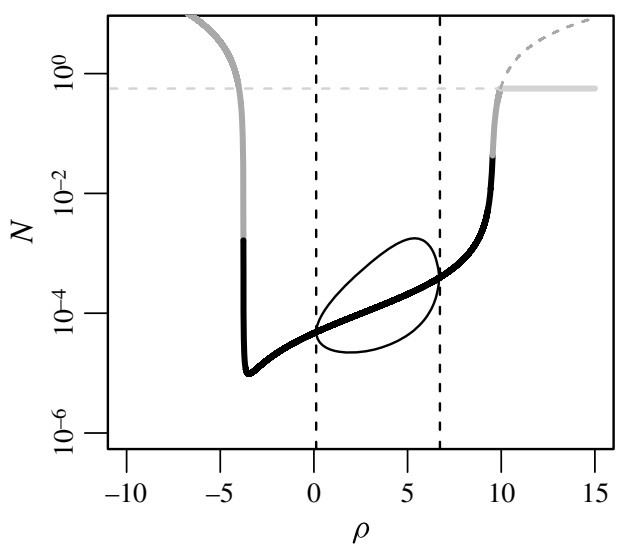

FIGURE 3. Bifurcation diagram at $L \approx 22$ days. Thick solid lines denote a stable equilibrium and thin solid lines represent an unstable equilibrium. The colours of these lines denote distinct equilibria (black is host extinction, dark grey coexistence, and light grey disease extinction). The thin dashed vertical lines denote the boundary of the Hopf region, the thin solid black curve within this region marks the extent of the limit cycle, and the thick black line within this region marks the unstable equilibrium around which the limit cycle oscillates.

the amplitude of the oscillations remains relatively small. We would like to get a feel for the behaviour of limit cycles within the boundary of the Hopf curve, and not only in one cross-section of the region. We calculate the boundary of the Hopf region by numerically solving the conditions in (3.29) for case 4. We then repeatedly integrate the ODE system at parameter values in and around the Hopf region to converge upon the limit cycle (where it exists) and find its amplitude, which we define as the range of the total population $N$, along with its period. The initial condition is set at a point near the unstable equilibrium: near enough to find the limit cycle reliably, and far enough away that convergence is relatively swift.

To aid rapid convergence, after every 250 years of integration we attempt to estimate the position of a point on the limit cycle by fitting the local maxima, or peaks, in $S$ to an exponential function during the simulation process. We assume that these peaks will, from an initial peak $p_{0}$, converge on the limit cycle's final peak $p_{\infty}$ at an unknown exponential rate $k$ such that at $n$ peaks after the initial peak, the value of the current peak $p_{n}$ will be estimated by

$$
p_{n}=p_{\infty}+\left(p_{0}-p_{\infty}\right) e^{-k n}
$$

We use a least-squares optimization algorithm to estimate the best-fit parameters $p_{\infty}$, $p_{0}$ and $k$. If this algorithm converges successfully, we then use it to calculate an estimate for a new initial condition for the system in the next iteration; otherwise, we continue the process as normal. We also make the estimator adaptive by increasing the error tolerance by $10 \%$ after every iteration. The tolerance is initially set as a relative difference of $10^{-5}$ between the values of any consecutive peaks in the limit cycle in $S$. 

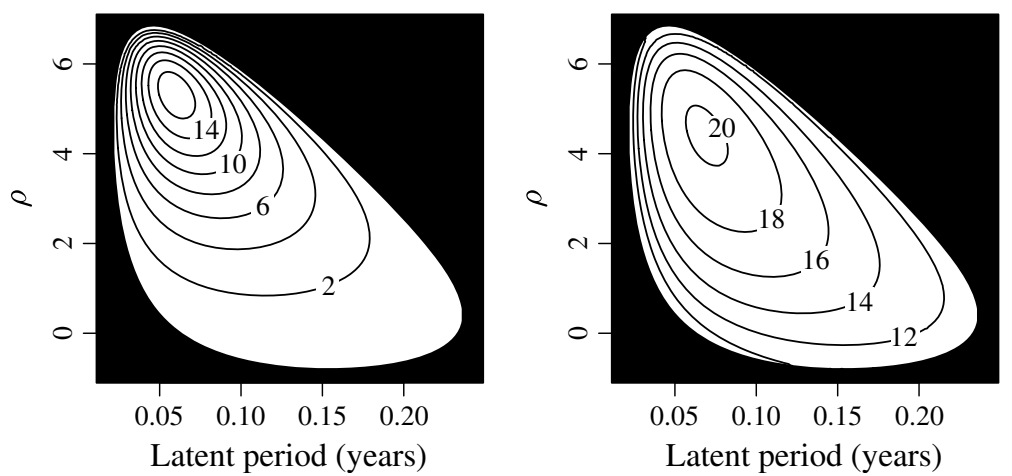

FIGURE 4. Contour plots of amplitude (in multiples of $\epsilon$, left) and period (in years, right) of the limit cycle. The black region describes the area of parameter space outside the Hopf region.

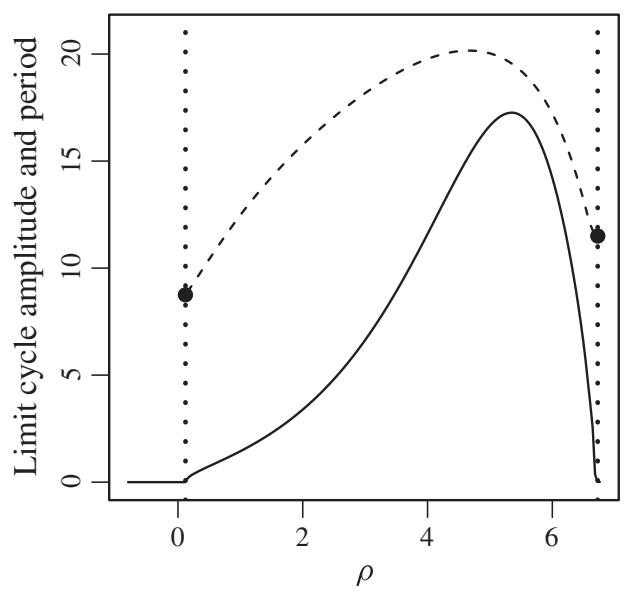

FIGURE 5. The amplitude in multiples of $\epsilon$ (solid line) and period in years (dashed line) of the limit cycle at $L \approx 22$ days, for varying values of $\rho$. The vertical dotted lines denote the boundaries of the limit cycle, and the black filled circles mark the analytical values for the zero-amplitude period at the boundaries.

Figure 4 shows the results. We can see that the limit cycle has a maximum amplitude of approximately $17.3 \epsilon$ at about $(L, \rho)=\left(0.06\right.$ years, 5.4 years $\left.^{-1}\right)$. The period of oscillation tends to increase with amplitude, but the two measures do not coincide: the maximum period of approximately 20.3 years does not occur at the same point as the maximum amplitude. The period also changes around the boundary of the Hopf curve, whereas the amplitude is necessarily zero at the boundary.

Figure 5 shows a vertical cross-section of the contour plot in Figure 4 holding $L$ constant at 0.06 years, or 22 days, in the region where the maximum amplitude was found, showing in more detail that the amplitude and period of the limit cycle have different behaviour. In the amplitude plot, the slope of the amplitude becomes vertical at the boundaries of the Hopf region on both sides, on a small scale to the left, and on 
a much larger scale to the right. This is expected, as the amplitude near the boundary of the Hopf region has been analytically shown by Guckenheimer and Holmes [8, pp. 150-152] and by Arrowsmith and Place [2, pp. 224-226] to be of order $\sqrt{x}$ where $x$ is the distance from the boundary.

At the boundary of the Hopf curve, the period of the zero-amplitude oscillation is equal to $2 \pi / \beta$, where the parameter $\beta$ is obtained from equation (3.28). At this boundary, two of the eigenvalues occur as the complex conjugate pair $\lambda= \pm i \beta$, and the period $2 \pi / \beta$ computed from (3.28) is indicated by black circles in Figure 5 at each of the two Hopf bifurcation points. The agreement with the numerical estimate of the period, indicated with a dashed line, gives confirmation of the accuracy of the computational results.

\section{Discussion}

In this paper, a mathematical model has been advanced for describing the dynamics of DFTD, based on the work of Beeton and McCallum [4]. This model is a specific case of the model framework described by Roberts and Jowett [18], and we have examined the biologically and mathematically interesting behaviours of the system in more detail. In particular, the existence of transcritical bifurcations between host extinction, coexistence and disease extinction, as demonstrated in Figure 2, gives evidence that disease suppression is theoretically feasible given sufficient removal effort.

Further, it quantifies the level of effort (3.16) required to achieve suppression under the model assumptions. As seen by points A and B of Figure 1, this level is extremely high, but the figure further shows that varying the latent period $L$ can affect the very nature of the problem: increasing it (or equivalently, decreasing $k=1 / L$ ) past a certain point removes the host extinction scenario entirely. Combinations of these two, and other, parameters may become relevant in the case of DFTD; in particular, recent fieldwork in the West of Tasmania [9] has suggested that a different strain of the disease may cause a lower mortality rate. We also surmise that such a strain may be slower growing, increasing the latent period of the disease, though no evidence yet exists to support such a hypothesis as the disease latent period is notoriously difficult to estimate. Point $\mathrm{C}$ then represents a disease with a one-year latent period and a sixmonth disease mortality time, as opposed to three months for our current estimate. To represent decreasing disease mortality by $2(\alpha=2, \rho=0)$ in point $\mathrm{C}$, we have kept $\alpha$ constant and instead reduced $\rho$ by $2(\alpha=4, \rho=-2)$.

Perhaps counter-intuitively, such a scenario would be no more amenable to disease suppression (increasing $\rho$ ) than point A. However, if the latent period were larger than expected, or disease evolution caused tumours to grow more slowly (point D), a much smaller removal effort would be required to avoid the extinction scenario (point E). These examples illustrate how changes in both devil and disease biology, via coevolution or by human interference, can change the stable equilibrium of the system. Such interference may have unexpected effects as avoiding extinction can 
be managed in two ways: by making disease mortality low enough that it is not a threat and coexistence is feasible (as may be possible in populations with a particular strain [9]), or by making it so high that it burns itself out without taking the entire population with it.

Having reasonable estimates of parameters is therefore vital to understanding the system properly, and this remains for future experimental work and analysis. Future modelling work will involve fitting this model to field data using Bayesian techniques (in preparation).

The asymptotic parameter $\epsilon$ was introduced in order to remove the singularity in the system of equations (2.1)-(2.4) where $N=0$. For the model to remain valid, the addition of $\epsilon$ should not introduce artificial dynamical behaviour on a large scale. Small-scale behaviour, however, is biologically irrelevant in this instance because any population that reaches such a low relative population will be doomed by stochastic effects in any case.

Equations (3.18) and (3.30) demonstrate that the stability of solutions, and by extension the location of the boundary of the Hopf curve, are at no point dependent on $\epsilon$. The only effect the size of $\epsilon$ has is on the amplitude of the host extinction steady state as defined in (3.13) and the amplitude of the limit cycles, which from Figure 4 appears to be of order $\epsilon$. The behaviour of the limit cycles, while mathematically interesting in the context of better understanding the system, is hence not of biological interest and does not affect the validity of the equations in a biological sense.

We can thus state that the system of equations appears to be a valid representation of host-disease dynamics under dynamical systems analysis. Extensions are made to this system by Beeton and McCallum [4] to take age structure and distribution of the latent period into account, and the results of this modelling are compared to field data collected concerning Tasmanian devils and DFTD. The models provided a reasonable fit to the data in most cases, suggesting that this kind of model is a reasonable first estimate of population and disease dynamics.

\section{Acknowledgements}

We are grateful to Clare Hawkins (Department of Primary Industry, Parks, Water and Environment), Rodrigo Hamede (University of Tasmania), Menna Jones (University of Tasmania), two anonymous reviewers and the editors for comments that helped to improve this paper. This work is supported in part by a Japanangka Errol West Indigenous Scholarship and a QANTAS Tasmanian Devil Research Scholarship awarded to Nick Beeton.

\section{References}

[1] R. M. Anderson and R. M. May, Infectious diseases of humans: dynamics and control (Oxford University Press, Oxford, 1991).

[2] D. K. Arrowsmith and C. M. Place, Dynamical systems: differential equations, maps, and chaotic behaviour (Chapman \& Hall, London, 1992). 
[3] N. D. Barlow, "A spatially aggregated disease host model for bovine Tb in New Zealand possum populations", J. Appl. Ecol. 28 (1991) 777-793; doi:10.2307/2404207.

[4] N. J. Beeton and H. I. McCallum, "Models predict that culling is not a feasible strategy to prevent extinction of Tasmanian devils from facial tumour disease", J. Appl. Ecol. 48 (2011) 1315-1323; doi: $10.1111 / \mathrm{j} .1365-2664.2011 .02060 . x$.

[5] O. Diekmann and J. A. P. Heesterbeek, Mathematical epidemiology of infectious diseases: model building, analysis and interpretation (Wiley, Chichester, 2000).

[6] L. Edelstein-Keshet, Mathematical models in biology, Volume 46 of Classics in Applied Mathematics (Society for Industrial and Applied Mathematics, Philadelphia, 2005).

[7] D. Fouchet, G. Leblanc, F. Sauvage, M. Guiserix, H. Poulet and D. Pontier, "Using dynamic stochastic modelling to estimate population risk factors in infectious disease: the example of FIV in 15 cat populations", PLoS One 4 (2009) 1-12; doi:10.1371/journal.pone.0007377.

[8] J. Guckenheimer and P. Holmes, Nonlinear oscillations, dynamical systems, and bifurcations of vector fields (Springer-Verlag, New York, 1990).

[9] R. Hamede, S. Lachish, K. Belov, G. Woods, A. Kreiss, A.-M. Pearse, B. Lazenby, M. Jones and H. McCallum, "Reduced effect of Tasmanian devil facial tumor disease at the disease front", Conserv. Biol. 26 (2012) 124-134; doi:10.1111/j.1523-1739.2011.01747.x.

[10] C. E. Hawkins, H. McCallum, N. Mooney, M. Jones and M. Holdsworth, Sarcophilus harrisii, IUCN Red List of Threatened Species, Version 2011.2, 2011, IUCN.

[11] H. McCallum, "Tasmanian devil facial tumour disease: lessons for conservation biology", Trends Ecol. Evol. 23 (2008) 631-637; doi:10.1016/j.tree.2008.07.001.

[12] H. McCallum, N. Barlow and J. Hone, "How should pathogen transmission be modelled?", Trends Ecol. Evol. 16 (2001) 295-300; doi:10.1016/S0169-5347(01)02144-9.

[13] H. McCallum and M. Jones, "To lose both would look like carelessness: Tasmanian devil facial tumour disease", PLoS Biol. 4 (2006) 1671-1674; doi:10.1371/journal.pbio.0040342.

[14] H. McCallum, M. Jones, C. Hawkins, R. Hamede, S. Lachish, D. L. Sinn, N. Beeton and B. Lazenby, "Transmission dynamics of Tasmanian devil facial tumor disease may lead to diseaseinduced extinction", Ecology 90 (2009) 3379-3392; doi:10.1890/08-1763.1.

[15] J. D. Murray, Mathematical biology: I. An introduction, 3rd edn (Springer-Verlag, New York, 2002).

[16] R Development Core Team, $R:$ A language and environment for statistical computing (R Foundation for Statistical Computing, Vienna, 2011).

[17] M. G. Roberts, “The pluses and minuses of $R_{0}$ ", J. Roy. Soc. Interface 4 (2007) 949-961; doi:10.1098/rsif.2007.1031.

[18] M. G. Roberts and J. Jowett, "An SEI model with density-dependent demographics and epidemiology”, IMA J. Math. Appl. Med. 13 (1996) 245-257; http://imammb.oxfordjournals.org/ content/13/4/245.

[19] R. T. Sterner and G. C. Smith, "Modelling wildlife rabies: Transmission, economics, and conservation", Biol. Conserv. 131 (2006) 163-179; doi:10.1016/j.biocon.2006.05.004.

[20] G. Wasserberg, E. E. Osnas, R. E. Rolley and M. D. Samuel, "Host culling as an adaptive management tool for chronic wasting disease in white-tailed deer: a modelling study", J. Appl. Ecol. 46 (2009) 457-466; doi:10.1111/j.1365-2664.2008.01576.x. 